\title{
Forecasting of Solar Power using Quantum GA - GNN
}

\author{
D.K. Chaturvedi \\ Department of Electrical Engineering \\ DEI, Dayalbagh, Agra, U.P., India
}

\begin{abstract}
Artificial Neural Network has been popularly used for forecasting purposes over the past. There are some innate problems in neural network such as indefinite configuration, architecture, and learning issues, etc. To vanquish these problems, Generalized Neural Network (GNN) has been used. This paper illustrates the development of Quantum GA-GNN method for forecasting of solar photovoltaic system power output. The actual data has been collected from the solar system installed at the rooftop of the University building and processed. The forecasting models also developed using Artificial Neural Network (ANN), and the results are compared.
\end{abstract}

\section{Keywords}

Solar Power Forecasting, Quantum, Genetic Algorithm, GNN, Neural Netowrk.

\section{INTRODUCTION}

In recent years, lack of adequate transmission capacity, limitations in constructing new transmission lines and emerging electricity markets in developing countries have been the main driving forces behind the increased use of Distributed Generations (DGs), which permits small-scale generators to be installed at the distribution level of the power system close to the consumer sites [1]. Many DG systems employ renewable resources for electricity generation; therefore, help in mitigation of adverse environmental impacts of the fossil fuel-based centralized generation. Among renewable DG systems, solar Photo Voltaic (PV) solar systems have attracted considerable attention and investment in several countries. Despite its relatively high cost and low efficiency, the PV based energy generation is expected to significant progress and penetrate into the present power system.

A PV cell is the fundamental unit which generates the voltage that varies in the range of 0.5 to 0.8 volts depending on cell manufacturing technology used. This voltage generation cannot be of much use for commercial applications but when we look physically in a PV system the module that is available commercially; which can be further reconnected in series and parallel to get the desired voltage and energy levels [1]. The forecasting of solar PV system output involves enormous factors mainly depends on the knowledge about the path of sun, the weather conditions, operating environment, the light scattering processes and the quality of solar PV modules in DG plant. The PV power output depends on the incoming solar energy into the form of light and on the solar panel characteristics. The Forecasted information of solar PV output is essential for an efficient use for the system, the management of the electricity grid and for solar-energy trading. The variability of solar power generation for consecutive four days is shown in Fig. 1. This shows that the power generation is not constant, but it varies depending on operating conditions (like operating temperature, dust deposition and shading on solar panels, etc.). This distributed solar power generation not only improves the power problems of developing countries, but also reduce carbon footprints, transmission losses and load frequency problems. This generation is done in two ways; namely islanding mode or connected with the conventional grid system. For its optimal usage and planning, forecasting of solar power generation is very important. The solar power forecasting is an important factor in efficient and optimal utilization of storage facilities available [2, 22]. Many researchers already developed the forecasting models using different methods. These forecasting methods mainly classified based upon the type of data i.e. time-series data or image. The statistical methods which use time-series data such as ARMA, ARIMA models [3], neural networks [4-8], NNs combined with wavelets [9-12].

The present work deals with the solar PV output prediction using ANN. The simple neural approach has certain problems such as issues related to the selection of network structure /architecture, its training, and the optimal use of training data. To overcome some of these problem's Quantum GA-GNN is proposed in this paper. The GNN developed earlier and used for short term electrical load forecasting and compared with ANN [13-16]. To improve the GNN training Quantum GA is used in this paper. The paper is divided into five sections besides introduction (as section- I). The section - II deals with the collection of PV output data for training and testing. Section - III and IV present the development of ANN and quantum GA-GNN models for forecasting of solar PV output. Section V compares the forecasted outputs and discusses the results. Finally, the work is concluded in last section.

\section{COLLECTION OF TRAINING DATA}

The training data for neural network has been contrived after acquisition of power output of solar Panel through a data logger which is prepared in the Dept. of electrical Engineering, Faculty of Engineering, Dayalbagh Educational Institute (Deemed University), Agra, India. The power output has been recorded at every one minute, and the pattern is shown in the Figure 1. This raw data is processed to remove unwanted sensor noise and then compose the training data for neural network. 


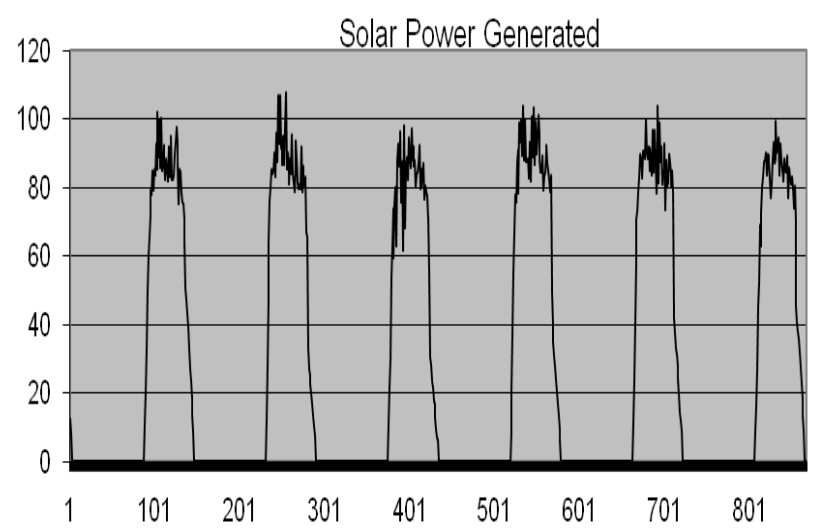

Fig. 1. Power output of Solar Panel for six days

The past four data is considered as input and the fifth data is taken as output. The training pattern is consisting of input vector $\mathrm{X}$ and output vector $\mathrm{Y}$ which is forecasted power 10minutes ahead as given in equation (1).

Input Vector $\mathrm{X}=[\mathrm{x}(\mathrm{t}) \mathrm{x}(\mathrm{t}-\mathrm{T}) \mathrm{x}(\mathrm{t}-2 \mathrm{~T}) \mathrm{x}(\mathrm{t}-3 \mathrm{~T})]$

Output Vector $\mathrm{Y}=[\mathrm{x}(\mathrm{t}+\mathrm{T})]$

Training Pattern $=\left[\begin{array}{ll}\mathrm{X} & \mathrm{Y}\end{array}\right]$

\section{FORECASTING MODEL USING}

\section{ANN}

In this section, artificial neural network (ANN) model is developed to project the power generated by Solar Photovoltaic system one time unit ahead. The ANN considered for this work is a three-layer architecture (namely, input layer, two hidden layer and output layer). The ANN configuration is shown in Table -1 .

Table - 1 ANN configuration

\begin{tabular}{|c|c|}
\hline Layer & Number of Neurons \\
\hline Input & 4 \\
\hline Hidden -1 & 22 \\
\hline Hidden - 2 & 10 \\
\hline output & 1 \\
\hline
\end{tabular}

All the neurons of ANN have summation as aggregation function and sigmoid as threshold function. The inputs were fed into Artificial Neural Network (ANN) and its performance is optimized (trained) using Levenberg Marquett algorithm. This trained ANN is then used for forecasting the solar power output of PV system.

Fig. 2 shows the structure of ANN and Fig. 4 represent the block diagram of ANN training using different learning algorithms such as LM or QGA.

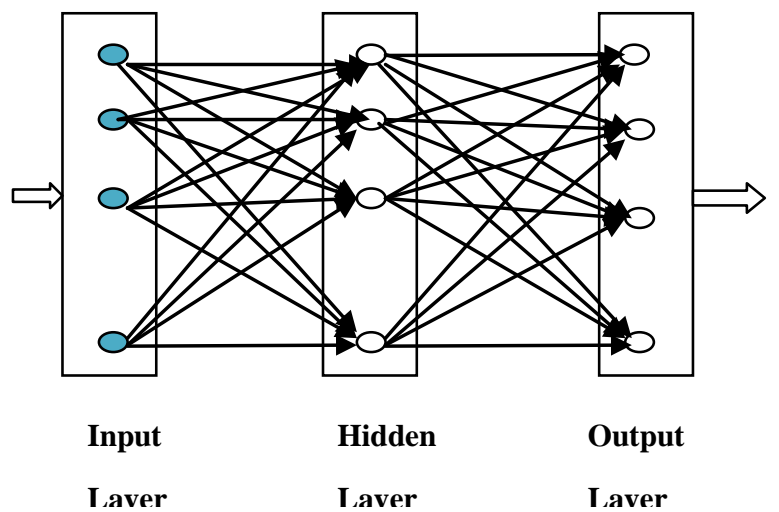

Fig.2 Network Architecture of ANN Model

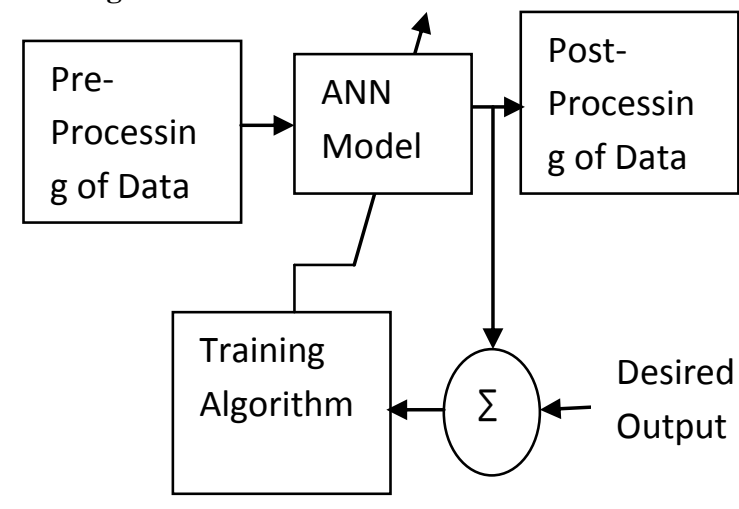

Figure: 3.Block diagram for ANN model development for solar forecasting

\section{DEVELOPMENT OF QUANTUM GA-GNN MODEL FOR FORECASTING}

\subsection{GNN model}

The GNN model used for solar power forecasting as shown in Fig. 4.

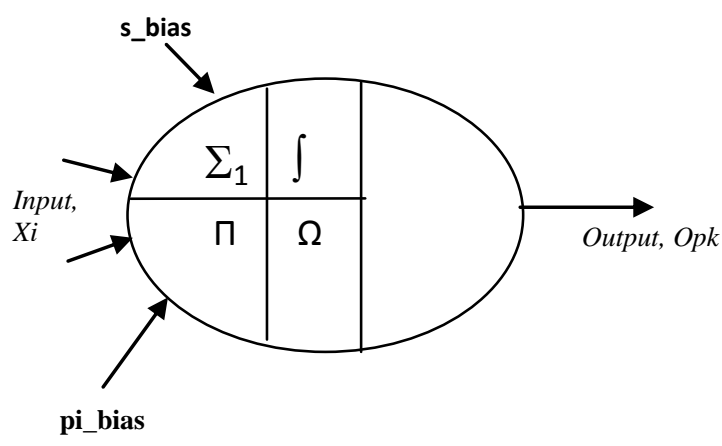

Fig. 4 Generalized neurons

The following steps are involved in the training of a summation type generalized neuron:

i. Forward Calculations

Step-1 The output of the $\sum 1$ part generalized neuron is

$$
O_{\Sigma}=\frac{1}{1+e^{-\lambda s^{*} s_{-} n e t}}
$$

where $s_{-}$net $=\sum W_{\Sigma i} X_{i}+X_{o \Sigma}$

Step-2 The output of the $\pi$ part of generalized neuron is

$$
O_{\Pi}=e^{-\lambda p^{*} p i{ }_{-} n e t^{2}}
$$

where $p i_{-}$net $=\prod W_{\Pi i} X_{i} * X_{o \Pi}$

Step-3 The final output of the generalized neurons can be written as

$$
O_{p k}=O_{\Pi} *(1-W)+O_{\Sigma} * W
$$


This output of generalized neuron is compared with the desired output to get error for the ith set of inputs:

$$
\text { Error } E i=(Y i-O i)
$$

\subsection{Quantum GA (QGA) for Training}

4.3 The development of quantum computing gives us a significant edge over classical computing in terms of time and efficiency. This is particularly useful for NP-hard problems such as ANN training. In QGA some of the features of quantum computing are implemented with the concepts of genetic algorithm [18-19]. The population of QGA is inspired by the concept of Q-bit in quantum computing. Q-bit is the building block of quantum computing [20, 21]. Q-bit can be represented in a two dimensional state space. A Q-bit individual is a string of $\mathrm{m}$ Q-bits, which is defined below.

$\left[\begin{array}{ccc}\alpha_{1} & \alpha_{2} \alpha_{3} & \alpha_{m} \\ \beta_{1} & \beta_{2} \beta_{3} & \beta_{m}\end{array}\right]$

(6)

Here $\alpha \mathrm{i}$ and $\beta \mathrm{i}$ are the probability of getting 0 and 1 respectively at position $i$. Also $|\alpha i| 2+|\beta i| 2=1$, for $i=1,2, \ldots \mathrm{m}$.

The Q-bit individual has the advantage that it can represent a linear superposition of states (binary solutions) in search space probabilistically. Thus, the Q-bit representation has a better characteristic of population diversity than other representations. A Q-gate is also defined as a variation operator of QGA to drive the individuals toward better solutions. The following rotation gate is used as a Q-gate in QGA, such as

$U\left(\Delta \phi_{i}\right)=\left[\begin{array}{cc}\cos \left(\Delta \phi_{i}\right) & -\sin \left(\Delta \phi_{i}\right) \\ \sin \left(\Delta \phi_{i}\right) & \cos \left(\Delta \phi_{i}\right)\end{array}\right]$

where , $\Delta \phi i$, is a rotation angle of each Q-bit toward either 0 or 1 state depending on its sign should be designed in compliance with the application problem.

The flow chart of QGA- GNN training is shown in Fig. 5.

\section{RESULTS}

The ANN model is trained using LM - algorithm in Matlab ver. 7.2. The results of ANN training is shown in Figs. 6 9. Also QGA-GNN is trained using quantum genetic algorithm. The maximum and average fitness improvements are shown in Fig. 10 for different generations. Here filtered data is used for training and obtained good results. Similarly QGA-GNN also trained for non-filtered solar data and found that QGA-GNN still worked better. The above developed QGA-GNN models after training are used for forecasting of solar power output of PV panels and the results are shown in Fig. 11 and 13. The RMS error also shown in Table -2 .

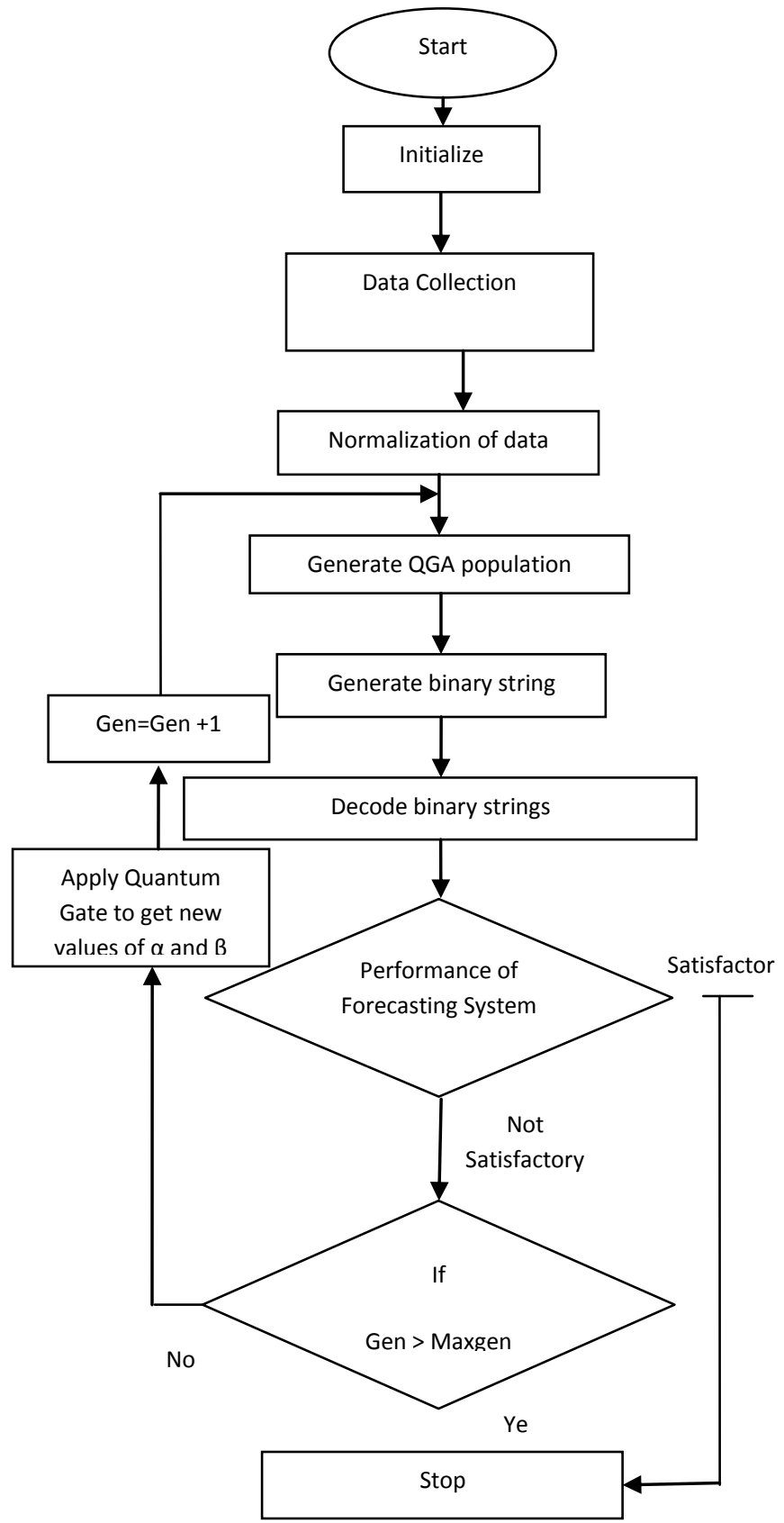

Fig. 5 Flow chart of QGA-GNN

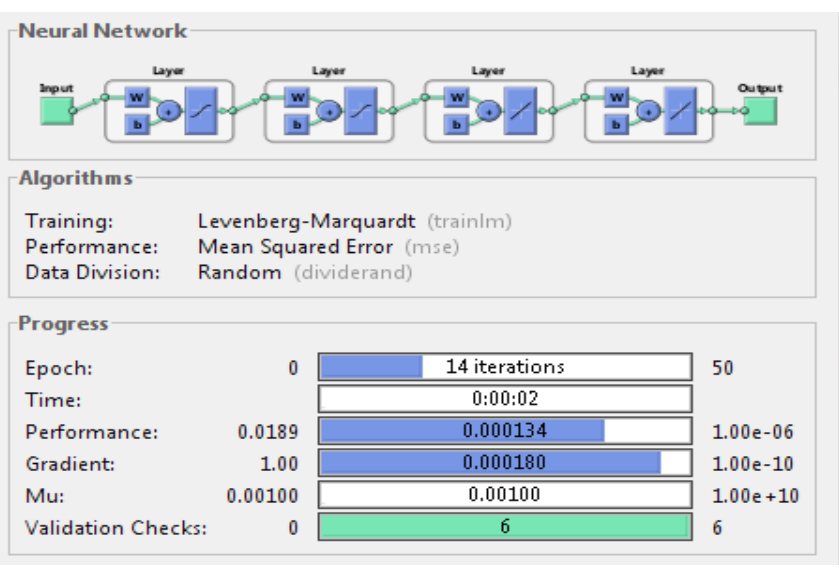

Fig. 6 ANN training 


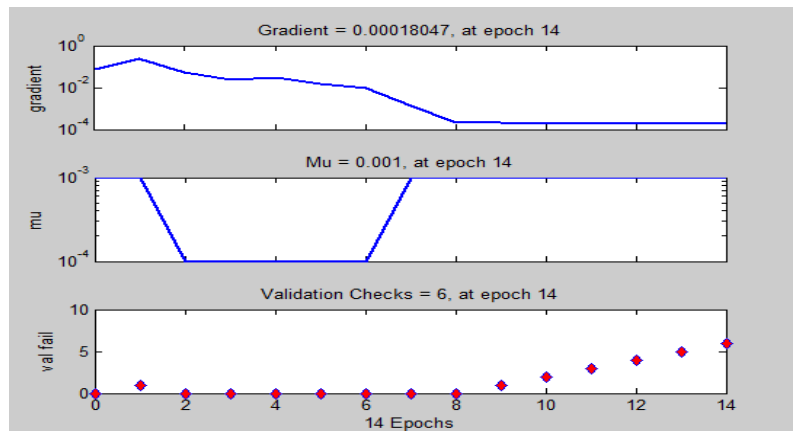

Fig. 7 Variation in training parameters during $L M-$ training of ANN.

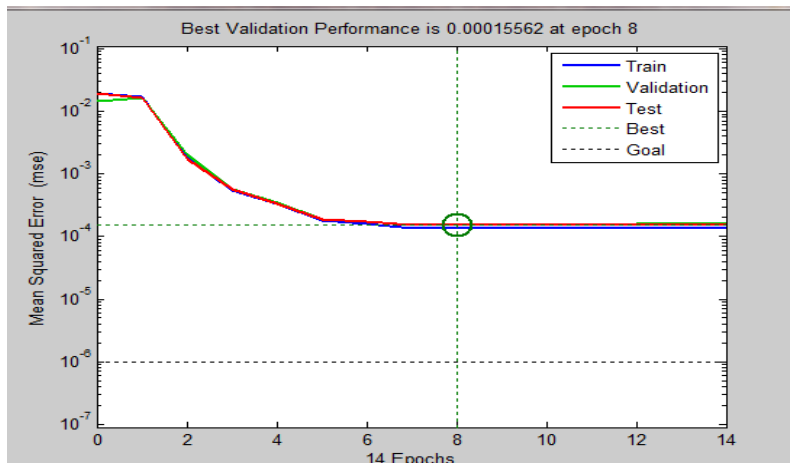

Fig. 8 Training Performance of ANN

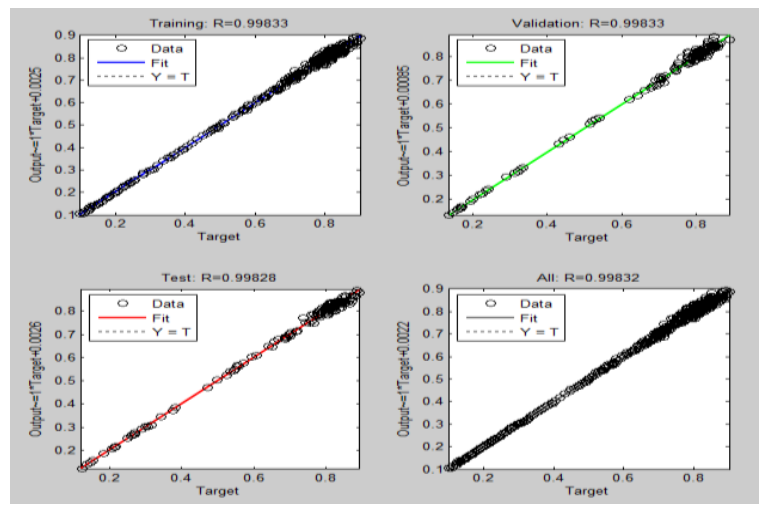

Fig.9 Regression plots after ANN training

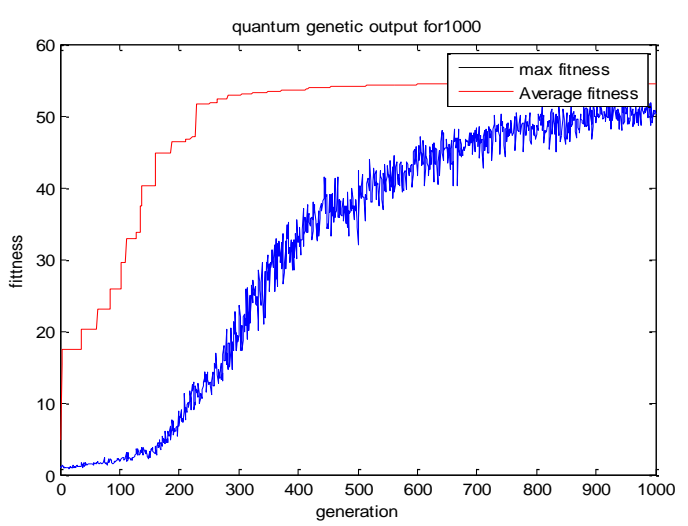

Fig. 10 Training Performance of QGA-GNN

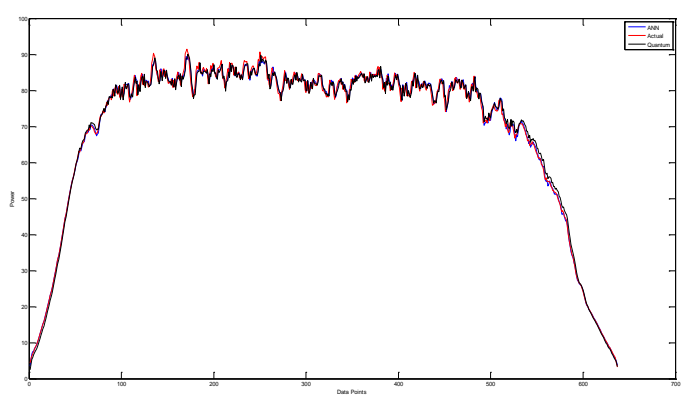

Fig. 11 Solar PV output forecasting using Quantum GAGNN and ANN

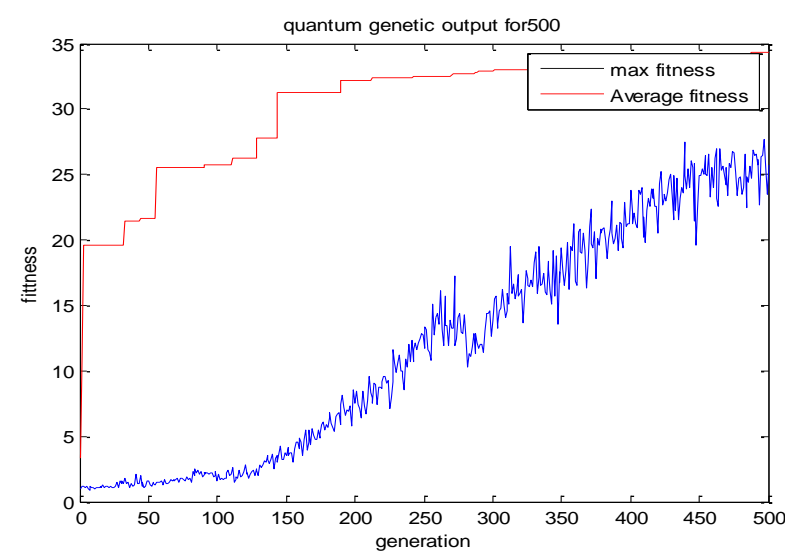

Fig. 12 QGA-GNN Training performance (Fitness)) for non-filtered mix data of 27 Feb and 23 Sept.2015

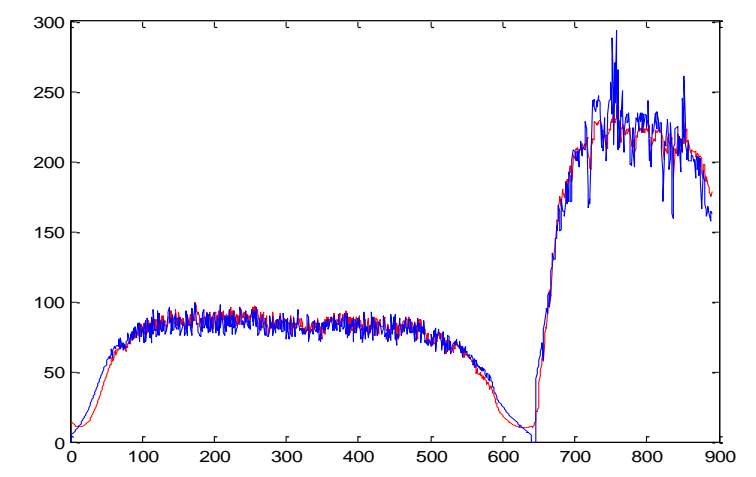

Fig. 13 QGA-GNN Training performance (prediction)) for non-filtered mix data of 27 Feb and 23 Sept. 2015

Table - 2 Performance comparison

\begin{tabular}{|c|c|c|c|c|}
\hline \multirow{2}{*}{ Models } & \multicolumn{2}{|c|}{ Filtered data } & \multicolumn{2}{c|}{ Non-filtered data } \\
\cline { 2 - 5 } & ANN & $\begin{array}{c}\text { QGA- } \\
\text { GNN }\end{array}$ & ANN & $\begin{array}{c}\text { QGA- } \\
\text { GNN }\end{array}$ \\
\hline $\begin{array}{c}\text { Training } \\
\text { performance } \\
\text { (rms error) }\end{array}$ & 0.000134 & 0.0001 & 0.00234 & 0.0005 \\
\hline $\begin{array}{c}\text { Testing } \\
\text { performance } \\
\text { (rms error) }\end{array}$ & 0.007 & 0.005 & 0.014 & 0.006 \\
\hline
\end{tabular}




\section{CONCLUSION}

The paper illustrates two forecasting models which are developed using artificial neural network (ANN) and Quantum based Genetic Algorith - Generalized Neural Netowrk (QGA-GNN) approaches for forecasting of the power output of solar PV system. The 'tansig', 'tansig', 'purelin' functions are used at two hidden and one output layers respectively in the feed forward neural network to develop ANN model. The LM- training is used for ANN forecasting model. Similarly, QGA-GNN model has been developed with single higher order neuron, trained and tested for the same data as used in ANN. The results are compared and the performance of QGA-GNN found better in terms of training and testing. Although, it is difficult to train the network for non-filter data as shown in results.

\section{REFERENCES}

[1] J. M. Guerrero, F. Blaabjerg, T. Zhelev, K. Hemmes, E. Monmasson, S. Jemei, M. P. Comech, R. Granadino, and J. I. Frau, "Distributed generation: Toward a new energy paradigm," IEEE Ind. Electron. Mag., vol.4, pp. 52-64, Mar. 2010.

[2] Koeppel, G., Korpas, M., 2006. Using storage devices for compensating uncertainties caused by nondispatchable generators. 2006 International Conference on Probabilistic Methods Applied to Power Systems, pp. $1-8$.

[3] Møller, J.J.K., Nielsen, H.A., Madsen, H., 2008. Timeadaptive quantileregression. Computational Statistics and Data Analysis 52 (3), 1292-1303.

[4] P. Bacher et al. 2009 Online short-term solar power forecasting, Solar Energy 83, pp. 1772-1783.

[5] Sfetsos, A., Coonick, A., 2000. Univariate and multivariate forecasting of hourly solar radiation with artificial intelligence techniques. Solar, Energy 68 (2), 169-178

[6] Chaturvedi, D.K. 'Modeling and Simulation of Systems using Matlab/Simulink", CRC Press, New York, 2010.

[7] Paras Mandal, Tomonobu Senjyu, Katsumi Uezato, and Toshihisa Funabashi, "Forecasting Several-HoursAhead Electricity Demand Using Neural Network," IEEE Conference on Power Syst., vol. 2,pp. 515-521, April 2004.

[8] Hocaoglu, F.O., Gerek, O.N., Kurban, M., 2008 Hourly solar radiation forecasting using optimal coefficient 2-D linear filters and feed-forward neural networks. Solar Energy 82 (8), 714-726.

[9] Cao, J., Lin, X., 2008. Study of hourly and daily solar irradiation forecast using diagonal recurrent wavelet neural networks. Energy Conversion and Management 49 (6), 1396-1406.

[10] Chaturvedi D.K., Sinha A. P. and Chandiok, A., Short Term Load Forecasting using Neuro-Fuzzy Wavelet Approach, Int. J. of Computing Academic Research (IJCAR), 2013(a), pp. 36-48.
[11] Chaturvedi D.K., Siddiqi A. H., Chandiok, A. and Agarwal S., Annual Rainfall Prediction using NeuroFuzzy and Wavelet Approach, Indian J. of Industrial and Applied Mathematics, Vol. 4(1), 2013(b), pp. 1632 .

[12] Chaturvedi D.K., Sinha Anand Premdayal, Malik O.P., Short Term Load Forecast using Fuzzy Logic and Wavelet Transform Integrated Generalized Neural Network, International Journal of Electrical Power and Energy Systems, Vol. 67 (2015) 230-237.

[13] Chaturvedi, D.K., Satsangi, P.S. \& Kalra, P.K, Fuzzified Neural Network Approach for Load Forecasting Problems, Int. J. on Engineering Intelligent Systems, Vol.9(1): 3-9, March 2001.

[14] Chaturvedi, D.K., \& Malik OP, A Generalized Neuron Based Adaptive Power System Stabilizer for Multimachine Environment, IEEE Trans. on Power Systems, Vol. 20(1): 358-366, Feb 2005.

[15] Chaturvedi, D.K., Man Mohan, Ravindra K. Singh \& Kalra PK, Improved Generalized Neuron Model for Short Term Load Forecasting, Int. J. on Soft Computing - A Fusion of Foundations, Methodologies and Applications, 8(1):10-18, April 2004.

[16] Chaturvedi D.K., Sinha A. P. and Chandiok, A., Short Term Load Forecasting using Soft Computing Techniques, Int. J. Communication, Network and System Sciences, Vol. 3, 2010, pp. 273-279.

[17] Heinemann, D., Lorenz, E., Girodo, M., 2006. Forecasting of solar radiation. In: Dunlop, E., Wald, L., Suri, M. (Eds.), Solar Resource Management for Electricity Generation from Local Level to Global Scale. Nova Science Publishers, New York, pp. 83-94.

[18] D. Ashlock, Evolutionary Computation for Modeling and Optimization, Springer, ISBN 0-387-22196-4, 2006.

[19] Kuk-Hyun Han and Jong-Hwan Kim, "Quantuminspired Evolutionary Algorithm for a Class of Combinatorial Optimization", IEEE transaction on Evolutionary Computation, Vol. 6, No. 6, December 2002.

[20] U.V. Vazirani, lecture notes on Qubits, Quantum Mechanics, and Computers for Chem/CS/Phys191, University of California, Berkeley, 2012. www.cs.berkeley.edu/ vazirani/.

[21] Chaturvedi D.K., Qamar Tanveer, Malik O. P., Quantum Inspired GA based Neural Control of Inverted Pendulum, International Journal of Computer Applications (0975 - 887) Volume 122 - No.23, July 2015.

[22] D.K. Chaturvedi, Rahul Saraswat, Shashank Sharma, "Modeling and Simulation of Solar Photovoltaic System to Study various Operating Conditions", International Journal of Electronics, Electrical and Computational System IJEECS ISSN 2348-117X, Volume 4, Special Issue March 2015. 